\title{
Real Time Intelligent Traffic Light System
}

\author{
VIJAY PRASAD ${ }^{1}$, UWAIS ALI ${ }^{1}$, MANSOUR H. ASSAF ${ }^{1}$, SUNIL R. DAS ${ }^{2,3}$, and SATYENDRA N. \\ BISWAS $^{4}$ \\ ${ }^{1}$ School of Engineering \& Physics, The University of the South Pacific, Suva, FIJI ISLANDS \\ assaf_m@usp.ac.fj \\ ${ }^{2}$ Department of Computer Science, College of Arts and Sciences, Troy University, Montgomery, \\ AL 36103 USA \\ ${ }^{3}$ School of Information Technology and Engineering, Faculty of Engineering, University of Ottawa, \\ Ottawa, ON K1N 6N5, CANADA \\ ${ }^{4}$ Department of Electrical and Electronic Engineering Ahsanullah University of Science and \\ Technology, \\ Dhaka 1208, BANGLADESH
}

\begin{abstract}
- with the increasing vehicle ratio on our roads, a lot of factors such as pollution, time constraints and environmental factors need to be addressed. One main issue to be addressed is traffic congestion during the peak hours. This issue affects drivers in numerous ways including loss of productive working hours by lining up in traffic queue. It also leads to loss of natural resource such as fossil fuel used by the vehicle engine while the car is running but lining up in traffic.

In this paper, we propose an intelligent sensor-based traffic light control system. To analyze and compare performance, the system was implemented in MATLAB/SIMULINK and simulation results demonstrate the feasibility and high accuracy of the proposed intelligent traffic light system.
\end{abstract}

Keywords—Sensors, traffic light, tracking, sensors, Global Positioning System

Received: August 22, 2019. Revised: February 14, 2020. Accepted: February 28, 2020. Published: March 4, 2020.

\section{Introduction}

The very occurring urbanization from rural localities in the gaze for a better life has led to a massive growth in the urban population and caused a great boost to the infrastructure industry. One of the overstretched infrastructures is the roads, a condition that came in existence with the increase in traffic [1]. With the high speed life that is being practiced today, traffic congestion is becoming an urgent issue to discuss and avoid. Traffic congestion leads to a decrease in the individual productivity and in that way of the society, as most of the productive working hours are wasted in traffic lines. The higher number of street vehicles, the limited road infrastructure and the absurd dissemination of the signaling system leads to the frequent chaotic congestion in today's roads. Indirectly, traffic flow congestion leads to pollution as well, as the engine of the vehicles remain in majority of the case and a large amount of natural resource such as petrol and fuel is consumed without any useful outcome [2].

Another contributing factor for the traffic congestion problem in today's modern society is the timing mechanism for the traffic light system. Today's traffic light systems are majority timer based that is, all lights at an intersection will remain green and red for equal time interval simultaneously. Therefore, to reduce these problems significantly, a new mechanism such as intelligent based traffic light systems needs to be implemented by introducing Infrared sensor based automation techniques in the traffic lights system. This paper proposes an intelligent traffic light system, and presents performance analysis.

An efficient and intelligent traffic lights system is being developed, as a solution for optimized road vehicle traffic flow and congestion avoidance.

The traffic light system is designed as such that it will automatically change its light to the other as the traffic increases to a particular light. Through sensing the traffic there would not be traffic conjunction on any particular way but a traffic flow.

The main objectives are to:

- Propose a mathematical model and architecture design for the smart traffic light system.

- Run computer visual simulation of the system for different scenarios.

- Implementation and test the system. 
In this paper, we propose model and implementation of an intelligent sensor-based traffic light control system. In the next paragraph, we present key findings from a literature review on the application of tracking marine vessels.

\section{Background}

The increasing speed of life, has increase the traffic congestion which is an issue in our day to day activities. It's the major cause now which can bring down the production of each person and thereby, the society as lots of work hours are being wasted waiting at the traffic signal. The increase in the volume of cars, the inadequate infrastructure and the irrational distribution of the signaling system are the main factor of this chaotic congestion [1].

The environment is also indirectly affected by this issue uprising as the pollution level increases as the engines remain. In most of cases, a big number of natural resources in forms of petrol and diesel is used without any positive outcome [2].

The highly increase in the population of our towns and cities, the busy nature of the roads and need to increase one's time, there comes a need for more dynamic, system design and efficient traffic manageable designs which seeks to overcome unnecessary congestion and lawlessness causes from undue delays [3].

There is a need to comprehend the function of the traffic signals so that there is improvement in the driving habits by controlling the speed in order to decrease the number of traffic accidents [3][4].

The more the number of drivers know the traffic operation the less frustrated they are going to be while waiting for the change in the state. The main aim will be to develop and implement the smart traffic light signal to overcome the waiting time of each lane of the cars and also to increase the total number of cars that can cross an intersection given the mathematical function to count the waiting time [4].

Under the conventional method, the traffic lights are set on the fixed timer delays for a different lane. The following lights are cycled while changing from one signal to the other signal creating the unwanted and congestion [1]. This method is currently used in Fiji and should be upgraded as it leads to many issues. This technique is very old and will not be able to cope with the current population density.

We are proposed an optimized design and implementation of an intelligent traffic light system using an ultrasonic sensor [6]. This sensor is used to detect the distance of the vehicles and allows the traffic to flow if traffic lane busy. The system will enhance environmental safety and improve time constrains on the roads. Therefore, it is an efficient way of to control traffic in an optimized and efficient manner. System architecture and modeling are briefly described in the next paragraph.

\section{Methodology}

The proposed solution is to build a smart traffic light prototype that optimizes traffic congestion using sensors. A thorough extensive study was done in order to understand the interaction between hardware and software system components. Aaccurate data is collected from current functional traditional traffic light systems. Figure 1 shown below presents the system architecture.

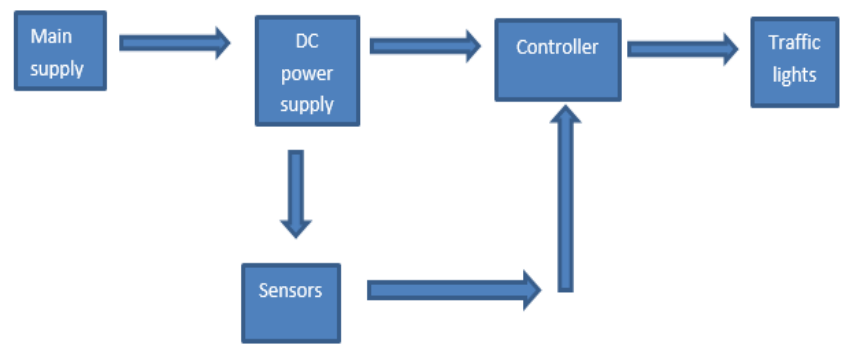

Fig. 1. Hardware implementation.

The above block diagram describes a high level overview of the hardware implementation of the system. A $240 \mathrm{~V}$ $\mathrm{AC}$ is the main power supply which gets converted to $5 \mathrm{~V}$ DC by the DC power source that further powers the controller and the sensor arrays [4]. The sensors deliver input to the controller which further performs logical operations in order to power the traffic lights. The circuit needs to be finalized and the schematic will be printed onto a PCB board on which all components will be soldered.

We have used Ultrasonic sensors for traffic and vehicle detection. The time taken by the ultrasonic sensor are pulse to leave the sensor, it bumps off the surface, and return to its location which is the sensor. As the distance taken is the full length of the time travel but we only need half of its time, so we divide the time by two. The speed variable which is used by the sensor is the speed of the sound waves. As the speed of sound changes in the 
air due to reasons such as temperature and humidity. In order to get the accurate distance calculated, we need to consider the ambient temperature and humidity.

Distance that sound travels $=$ Speed of sound in Medium

* Time that sounds travel.

For instance, only half a distance travelled by the sound wave is taken between object and the sensor.

Distance between sensor and object $=0.5 *$ Distance that sound travels.

The formula for the speed of sound in air with temperature and humidity accounted for is:

For the ultrasonic sensor the time is taken for only half a signals, as we only need half.

Hence, the time taken is time / 2 .

Distance $=$ Speed $*$ Time $/ 2$.

Speed of sound at sea level is $=343 \mathrm{~m} / \mathrm{s}$ or $34300 \mathrm{~cm} / \mathrm{s}$.

Thus, Distance $=171.5 *$ Time.

There will be four ultrasonic sensors interfaced with the Arduino board. These sensors will be read by Arduino and then, it will calculate the distance between the sensor and the vehicle.

The total time it takes for the ultrasonic wave to propagate, hit the object, and then return signal back to the sensor. Therefore, we divide time by two [2].

$\mathrm{s}=(\mathrm{t} * 0.034) / 2.0$

Figure 2 depicts the normal operation of Ultrasonic sensor.

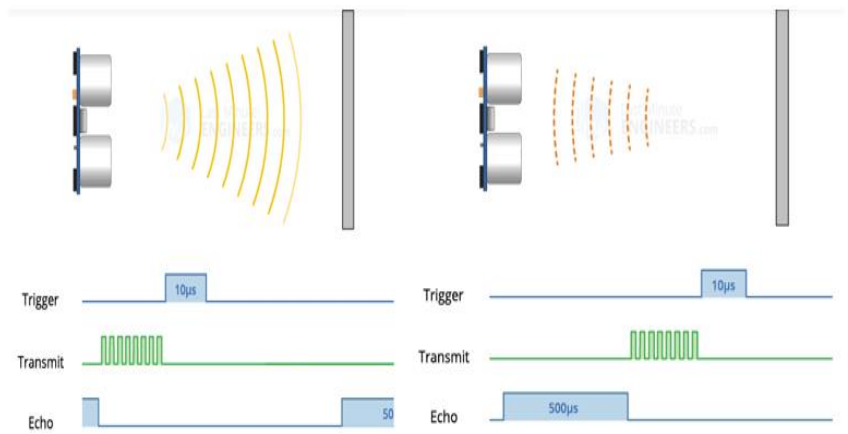

Fig. 2. Operation of Ultrasonic sensor [1].

Matlab and Simulink software will be used for the software implementation of this project. The FPGA software will be used for designing once the hardware implementation is complete.

\section{System Modeling}

The proposed system receive info from sensors in realtime. The system processes info, generates and interprets results.

The conventional method of traffic flow control is implemented with the fixed timers, which generates the operation cycle for a fixed time interval. Traffic flows are so inefficient and leads to traffic congestion and frustrations build up for many drivers, leading to accidents. For instance, if a timer is set for a green light is $30 \mathrm{sec}$ and yellow light is $5 \mathrm{sec}$, then to make one cycle for a signal will take 1.75 minutes to complete, leading to inefficiency of traffic vehicle flow. Figure 3 shows the conventional method of traffic control.

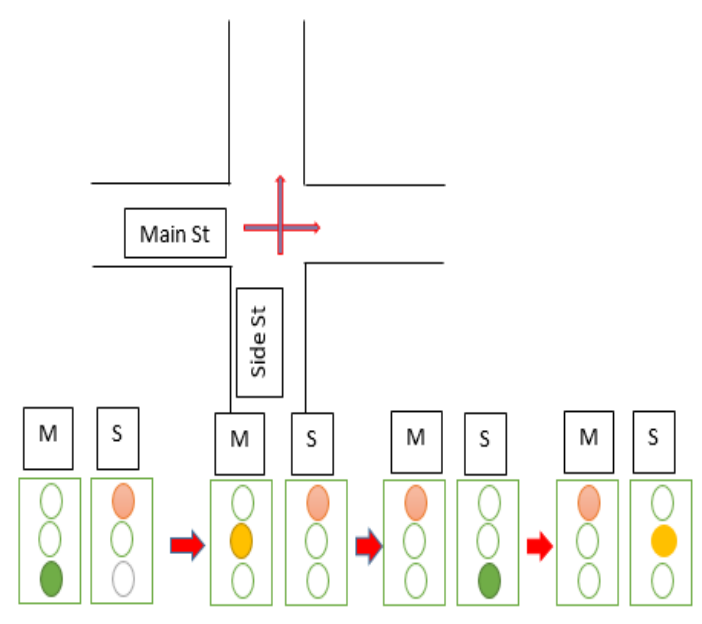

Fig. 3. Conventional traffic control. 
Table 1 shown below the traffic light state changes according to received inputs from the sensors. Table describes two lanes traffic behavior, going north and east.

Table 1. Two lanes traffic behavior.

\begin{tabular}{|c|c|c|c|c|c|}
\hline Input & & 00 & 01 & 10 & 11 \\
\hline $\begin{array}{l}\text { GO } \\
\text { North }\end{array}$ & 100001 & GON & $\begin{array}{l}\text { Wait } \\
\mathrm{N}\end{array}$ & GO N & Wait N \\
\hline $\begin{array}{l}\text { WAIT } \\
\text { North }\end{array}$ & 100010 & GOE & GOE & GO E & GO E \\
\hline $\begin{array}{l}\text { GO } \\
\text { East }\end{array}$ & 001100 & GO E & GO E & Wait E & Wait E \\
\hline $\begin{array}{l}\text { WAIT } \\
\text { East }\end{array}$ & 010100 & GO N & GO N & GO N & GO N \\
\hline & & $\begin{array}{l}\text { NO } \\
\text { Cars }\end{array}$ & EAST & $\overline{\mathrm{NORTH}}$ & BOTH \\
\hline
\end{tabular}

Tables 2 and 3 show different combinations and input/output and the associated states.

Table 2. Input/state combinations.

\begin{tabular}{|ll|}
\hline State & input \\
\hline No cars & 00 \\
\hline East cars & 01 \\
\hline North Cars & 10 \\
\hline Cars on both RD & 11 \\
\hline
\end{tabular}

Table 3. Output/state combinations.

\begin{tabular}{|ll|}
\hline State & Output \\
\hline Cars Going North & 100001 \\
\hline Stop North Cars & 100010 \\
\hline Cars Going East & 001100 \\
\hline Stop East Cars & 010100 \\
\hline
\end{tabular}

Input data from sensors are generated by sensing car traffic flow on the particular lane. Received sensor data input is used to update system states in an automatic manner to avoid traffic congestion.

\section{System Implementation}

The proposed smart traffic light system hardware implementation and various components are shown in figure 4. The main part of the project is the including the Arduino mega 2560 controller that reads signals from the HC-SR04 ultrasonic sensors and calculate the distance between vehicles and the traffic lights in order to optimize traffic flow and control traffic congestion.

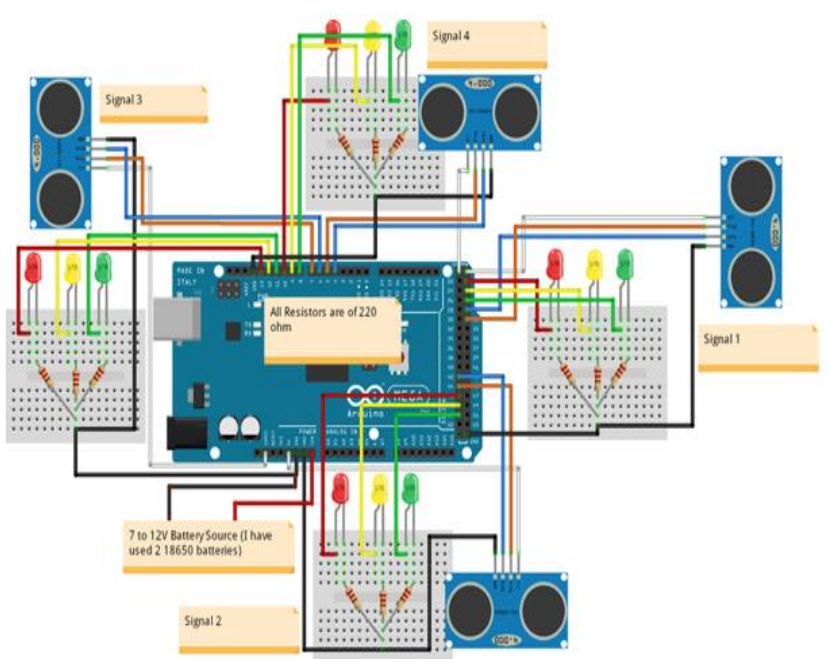

Fig. 4. Sensor-based 4-way traffic light system [8].

Proteus with Arduino software's was used to implement the software circuit design. The circuit was constructed in Proteus and its code was written in Arduino. The hex file was uploaded onto the microcontroller in Proteus.

Figure 5 shows the circuitry of a 4 way traffic light control system where every lane with be given fixed equal time for the traffic light to remain green. After the time is complete for a particular light at green, traffic light will change to yellow for a short period before it changes to red. Now all traffic lights will remain red and that represents that it is time for pedestrian crossing.

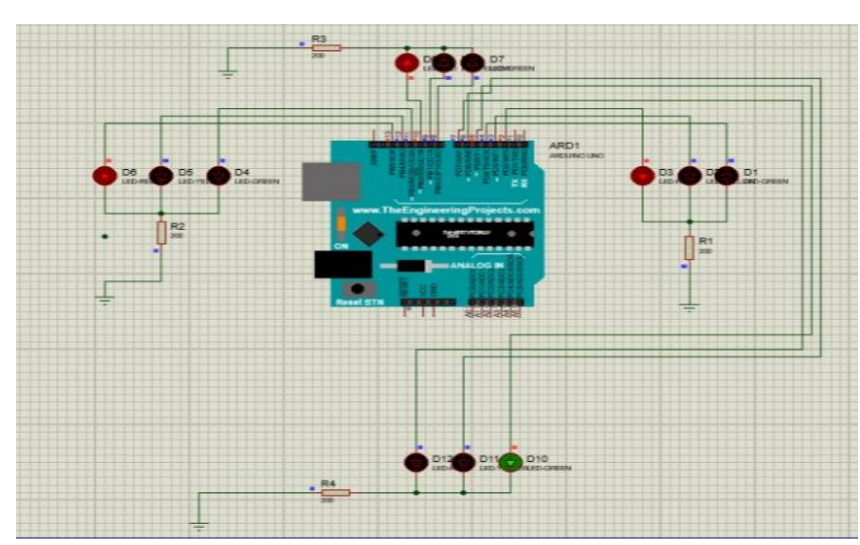

Fig. 5. 4-way traffic light system. 
Figure 6 shown below depicts a 4-way sensor based traffic light control system. The system is made up of 4 infrared sensors. Each of the four sensors is connected to a particular traffic light. Each of the traffic lights represents a pre-defined the road and it is denoted by a given direction.

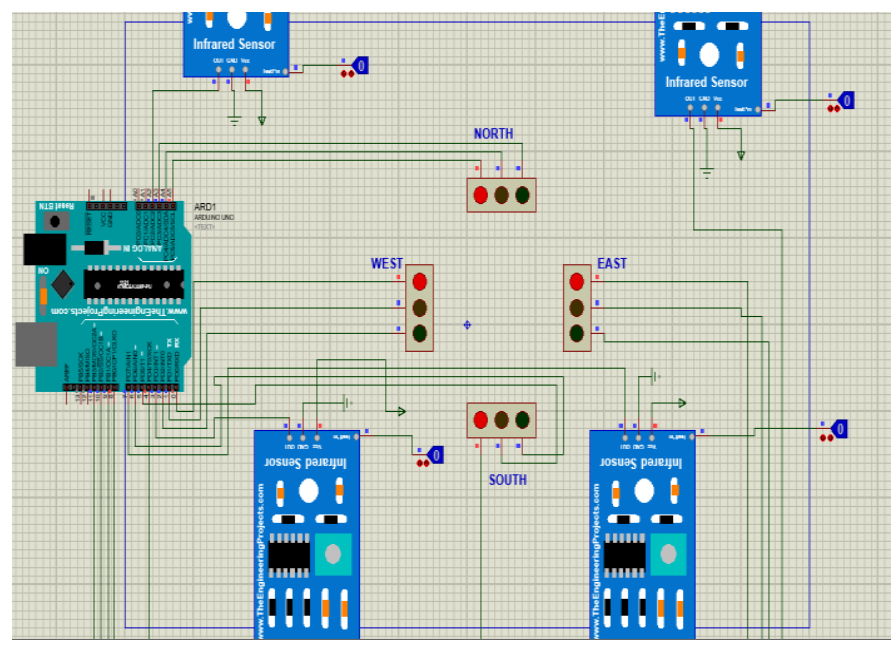

Fig. 6. Scenario 1: 4-way traffic light system.

Two logic states 0 and 1 are used in computer simulation to represent traffic density. The sensor state 0 logic represents no traffic follow and therefore pedestrian crossing is ON. However, the sensor state 1 represents a traffic situation where therefore pedestrian crossing is OFF and traffic light from red to green while the rest of the lights remain red.

Figure 7 and 8 show various scenarios and the transition of traffic light from red to yellow and then to green. When the logic state for the each side roads changes from 0 to 1 , the east side roads traffic light changed to green allowing traffic to flow only from the east direction.

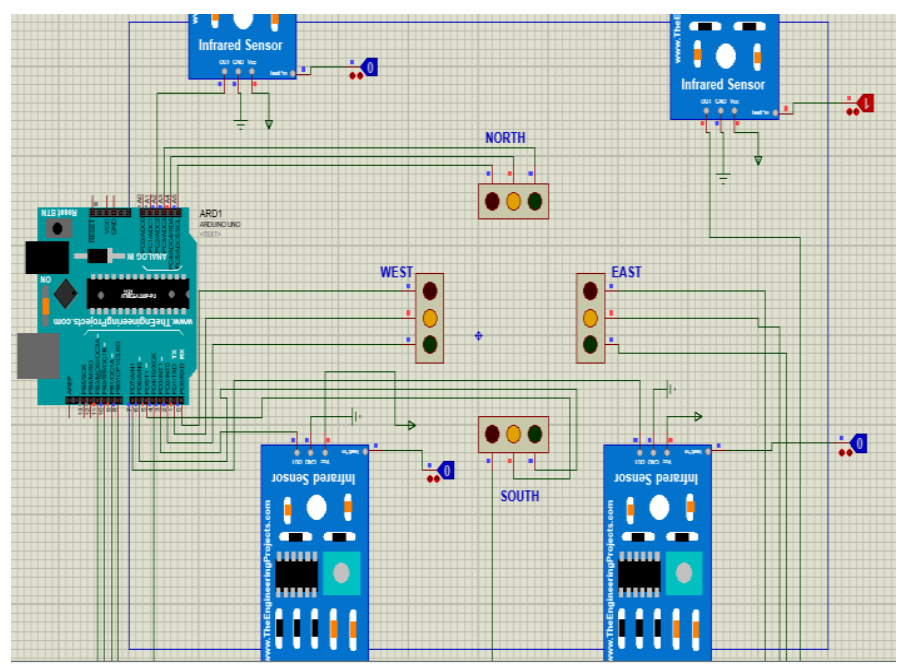

Fig. 7. Scenario 2: 4-way traffic light system.

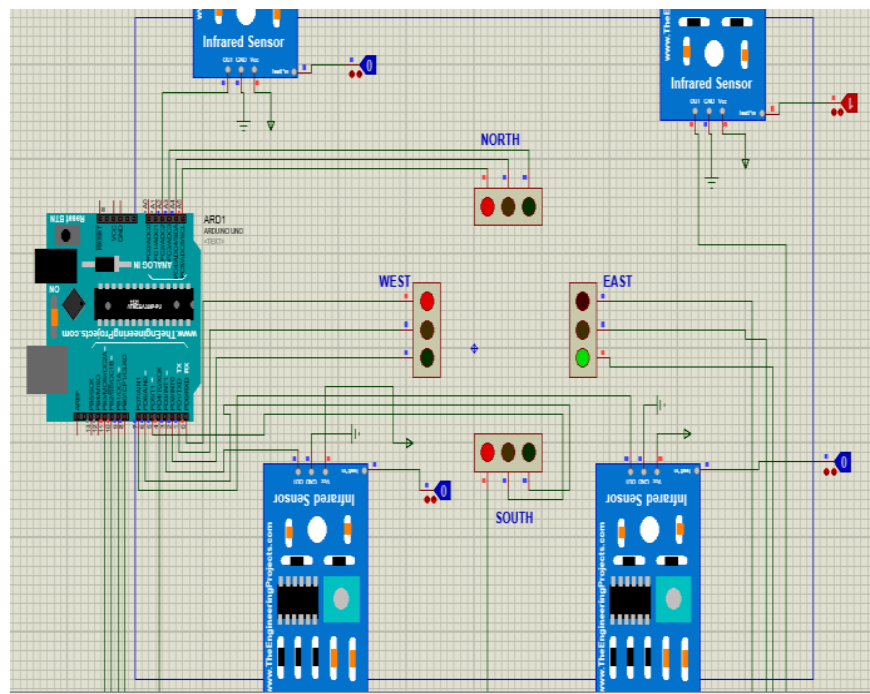

Fig. 8. Scenario 3: 4-way traffic light system.

In a situation where there is higher traffic density elsewhere, the sensor will switch logic from 0 to 1 and the traffic light will change from red to green after a predetermined time delay. Time delay is introduced to avoid car collision.

A well-planned traffic system is needed to avoid road accidents and give chances to emergency vehicles to pass through without any problems. The major challenge is to estimate traffic density at rush and not rush hours, therefore we conducted roads surveys at different times in various weather conditions in order to measure optimal system parameters. In the next section we present concluding remarks.

\section{Conclusion}

This paper discusses the design, implementation and validation of an intelligent traffic light system based on input data gathered from sensors. Computer simulation and actual prototype were used to validate the proposed design and to show its effectiveness. Obtained results show that the proposed system is promising and that it is effective in controlling road traffic flow and easing traffic congestion. We plan to extend this work further by applying other techniques and adding intelligence to the system controller to better optimize performance. 
References:

[1] M. H. W. M. H. A. Albagul, "Design and Development of Sensor Based Traffic Light System,"American Journal of Applied Sciences , vol. 3, 2006.

[2] S. D. N. N. S. M. Ashwini Basavaraju, "VEHICLE DENSITY SENSOR SYSTEM TO' MANAGE TRAFFIC," International Journal of Research in Engineering and Technology, vol. 3, no. 3, 2014.

[3] A. K. B. B. M. I. A. A. A. R. Faruk Bin Poyen, "Density based traffic control," International Journal of Advanced Engineering, Management and Science, vol. 2, no. 8, 2016.

[4] T. M. Koji Hayama, "A FAR INFRARED VEHICLE SENSOR FOR A TRAFFIC SIGNAL CONTROL," ITS Development Department, Sumitomo Electric Industries, Ltd., Osaka, Japan.

[5] R. Burnett, "MaxBotix," 27 November 2017. [Online]. Available: https://www.maxbotix.com/articles/ultrasonic-orinfrared-sensors.htm. [Accessed 4 April 2019].

[6] "Last Minute Engineers," [Online]. Available: https://lastminuteengineers.com/arduino-sr04ultrasonic-sensor-tutorial/. [Accessed 2 April 2019].

[7] D. Nedelkovski, "HowToMechatronics" 2004 [Online]. Available: https://howtomechatronics.com/tutorials/arduino/ultr asonic-sensor-hc-sr04/. [Accessed 3 April 2019].

[8] I. G. O. Y. N. Udoakah, "DESIGN AND IMPLEMENTATION OF A DENSITY-BASED TRAFFIC LIGHT CONTROL WITH SURVEILLANCE SYSTEM," Nigerian Journal of Technology (NIJOTECH), vol. 36, no. 4, 2017.

[9] M. H. P. Mr. Yogesh Shinde, "Intelligent Traffic Light Controller Using IR Sensors for Vehicle Detection," International Advanced Research Journal in Science, Engineering and Technology, vol. 4, no. 2, 2017.

[10]. S. Goel and C. Gershenson, "Self-Organization in Traffic Lights: Evolution of 'Signal Control with Advances in Sensors and Communications," GE Global Research, New York, 2011.

[11] R. Sundar, S. Hebbar and V. Golla, "Implementing Intelligent Traffic Control System for Congestion Control, Ambulance Clearance, and Stolen Vehicle Detection," IEEE SENSORS JOURNAL, vol. 15, no. 2, 2015.

[12] R. Ghosh , D. Rasaily and I. Dey , "Auto Density , Sensing Traffic Control System using At89s52," International Journal of Engineering Trends and Technology (IJETT), vol. 32, no. 5, 2016.

[13] Deekshitha, M. Disha and Soumya, "Traffic Monitoring System Using IR Sensors," International Journal of Advance Research, Ideas and Innovations in Technology., vol. 3, no. 3, 2017.

[14] J. R. Latha and U. Suman, "Intelligent Traffic Light Controller," International Journal of Emerging Engineering Research and Technology, vol. 3, no. 3, pp. 38-50, 2015.

[15] O. D. Ese and O. E. Ighodalo, "An Intelligent System for Traffic Control in Smart Cities: A Case Study," American Journal of Artificial Intelligence , vol. 1, no. 1, 2017.

[16] M. Aqib, "Electronics Hobbyists," 2016. [Online]. $\quad$ Available: https://electronicshobbyists.com/ultrasonic-distancesensor-hc-sr04-arduino-tutorial-proximity-sensor/.

[Accessed 3 April 2019].
[17] "Modelling and R. Su, Y. Y. Zhang and C. Sun, Heterogeneous Traffic SystemS," IEEE, vol. 1, 2017. 